\title{
Constraining neutrino mass in dark energy dark matter interaction and comparison with 2018 Planck results
}

\author{
Hamid Reza Amiri ${ }^{1}$, Amin Salehi ${ }^{2, a} \mathbb{C}_{\mathbb{O}}$, Amir Hossein Noroozi $^{1}$ \\ ${ }^{1}$ Department of mathematics, Hamedan Branch, Islamic Azad University, Hamedan, Iran \\ ${ }^{2}$ Department of Physics, Lorestan University, Khoramabad, Iran
}

Received: 13 November 2020 / Accepted: 19 May 2021 / Published online: 31 May 2021

(c) The Author(s) 2021

\begin{abstract}
In this paper, we investigate the constraints on the total neutrino mass $\sum m_{v}$ in a cosmological model in which dark energy and neutrinos are coupled such that the mass of the neutrinos and potentials are function of the scalar field as $m_{\nu}=m_{0} \exp \left(\frac{\alpha \phi}{m_{p l}}\right)$ and $V(\phi)=m_{p l}^{4} \exp \left(\frac{-\lambda \phi}{m_{p l}}\right)$ respectively. The observational data used in this work include the type Ia supernovae (SN) observation (Pantheon compilation), CC, $\mathrm{CMB}$ and $\mathrm{BAO}$ data. We find that the neutrino mass is tightly constrained to $\sum m_{v}<0.125 \mathrm{eV} 95 \%$ Confidence Level (C.L.) and the effective extra relativistic degrees of freedom to be $N_{\text {eff }}=2.955_{-0.12}^{+0.11} 68 \%$ C.L in agreement with the Standard Model prediction $N_{e f f}=3.046$, matter-radiation equality, $z_{e q}=3389_{25}^{+24}$ (68\% C.L). These results are in good agreement with the results of Planck 2018 where the limit of the total neutrino mass is $\sum m_{v}<0.12 \mathrm{eV}(95 \%$ C.L., TT, TE, $\mathrm{EE}+$ lowE + lensing + BAO),$N_{e f f}=2.99_{-0.17}^{+0.17}$ (68\% C.L., TT, TE, EE + lowE + lensing $+\mathrm{BAO})$ and $z_{e q}=$ $3387_{21}^{+21}$ (68\% C.L TT, TE, EE + lowE + lensing + BAO).
\end{abstract}

\section{Introduction}

The accelerating expansion of the universe [1-4] is one of the must surprising discoveries in cosmology. Also the observations of Cosmic Microwave Background (CMB) anisotropies indicate that the universe is flat and the total energy density is very close to the critical one [5].

According to General Relativity, the dynamic of the universe is dominated by a new (dark) energy form with negative pressure. There are prominent candidates for $\mathrm{DE}$ such as the cosmological constant $[6,7]$ in which dark energy takes the form of a cosmological constant and dark matter is taken to be cold, in other words having an equation of state equal to zero. While $\Lambda \mathrm{CDM}$ fits the available data very well, it suffers from a number of issues that motivate the study of alter-

\footnotetext{
a e-mail: salehi.a@lu.ac.ir (corresponding author)
}

natives. These include the fine-tuning [7] and coincidence [8] problems. In addition, there are certain tensions between early and late-universe observations in $\Lambda \mathrm{CDM}$. The presentday expansion rate of the universe, $H_{0}$ and the growth of structure, quantified by $\sigma_{8}$, can be calculated using the bestfit $\Lambda \mathrm{CDM}$ parameters to cosmological data, including the $\mathrm{CMB}$. This gives rise to a smaller $H_{0}$ and a larger $\sigma_{8}$ than the results of local, late-universe measurements (for a recent discussion see Ref. [9].

A popular class of modifications to $\Lambda \mathrm{CDM}$ is quintessence $[10,11]$, in which the cosmological constant $\Lambda$ is set to zero and a scalar field $\phi$ is introduced whose dynamical properties produce a negative equation of state giving rise to the observed late-time accelerated expansion of the universe. Normally it is assumed that the scalar field does not interact with dark matter. However there is no reason why this must be the case, and the consequences of relaxing this assumption have been widely studied. See Ref. [12] with references therein and [13-17] for a discussion of recent research on interacting dark energy. The other candidates are phantom (field with negative energy) [18] that explains the cosmic accelerating expansion. Meanwhile, the accelerating expansion of universe can also be obtained through modified gravity [19], brane cosmology and so on [20-43]. On the other hand, to explain the early and late time acceleration of the universe. It is most often the case that such fields interact with matter; directly due to a matter Lagrangian coupling, indirectly through a coupling to the Ricci scalar or as the result of quantum loop corrections [44-48]. If the scalar field self-interactions are negligible, then the experimental bounds on such a field are very strong; requiring it to either couple to matter much more weakly than gravity does, or to be very heavy [49-52]. Unfortunately, such a scalar field is usually very light and its coupling to matter should be tuned to extremely to small values in order not to be a conflict with the Equivalence Principal [53]. The discovery of the accelerated expansion of the universe is 
also the main challenge for particle physics [54]. It requires new physics for the explanation of dark energy. Neutrinos were first shown to have mass in observations of neutrino flavour oscillations $[55,56]$, the presence of which demands that at least two of the neutrino states are massive [57]. While the attempts of the laboratory experiments of particle physics to measure the absolute masses of neutrinos, have always been facing great challenges [58-68], the cosmological observations are more prone to be capable of measuring the absolute masses of neutrinos [65-67], since massive neutrinos can leave rich signatures on the cosmic microwave background (CMB) anisotropies and the large-scale structure (LSS) formation at different epochs of the cosmic evolution [68]. Recently some studies have attempted to constrain the total neutrino mass $\sum m_{v}$ and as well as the effective number of relativistic degrees of freedom $\left(N_{e f f}\right)$ using cosmological observations [69-126] Also the cosmological consequences of interacting dark energy and dark matter have been widely studied [127-166], however, we are interested in consideration that the role of neutrino is prominent. In this work, we implement a cosmological model which proposed by [167] and extend by [168] to constrain total neutrino mass with observations. In this model dark energy and neutrinos are coupled such that the mass of the neutrinos is a function of the scalar field $m_{v}=m_{0} \exp \left(\frac{\alpha \phi}{m_{p l}}\right)$. The scalar field plays the role of dark energy and drives the late time accelerated expansion of the universe. The motivation of such consideration has been investigated by [168-177]. Here we consider a generalized model of [167] which allows both dark matter and neutrino interact with dark energy with different interacting couplings $\beta$ and $\alpha$. We constrain on $\left(\sum m_{\nu}, N_{e f f}, h, \Omega_{m}, \Omega_{b} h^{2}, \Omega_{c} h^{2}, \alpha, \beta, \lambda, \omega\right)$ using observational data data. The structure of the paper is as follows. Section 2 introduces the cosmological model explored here while Sect. 3 describes the methodology and the measurements exploited in our data analyses. Section 4 presents our results and we conclude the article in Sect. 5

\section{The model}

The expansion rate of the Universe is given by Hubble parameter $H=\frac{\dot{a}}{a}$, where $a$ is scale factor and an overdot denote cosmic time derivative. We assume a spatially-flat Friedmann-Robertson-Walker Universe filled with baryons $(b)$, radiation $(r)$, dark energy $(\varphi)$, dark matter $(\mathrm{dm})$ and neutrinos $(v)$. The baryons and radiation are regarded as noninteraction fields. The Friedmann equations which follow from Einstein field equations are as

$$
3 H^{2}=\rho, 2 \dot{H}+3 H^{2}=-p
$$

Where, $\rho=\rho_{b}+\rho_{r}+\rho_{d m}+\rho_{v}+\rho_{\varphi}$ and $p=p_{b}+$ $p_{r}+p_{d m}+p_{v}+p_{\varphi}$. From the conservation of the energy momentum tensor it follows the evolution equation for the total energy density:

$\dot{\rho}+3 H \rho=0$

The baryons are treated like dust $\left(p_{b}=0\right)$ and the barotropic equation of state for the radiation field is $\left(p_{r}=\frac{1}{3} \rho_{r}\right)$. Once both of them have no interaction with other components, the evolution equations for their energy densities are :

$\dot{\rho}_{b}+3 H \rho_{b}=0$

$\dot{\rho}_{r}+4 H \rho_{r}=0$

respectively. Since the dark energy is modeled as a scalar field $\phi$ its energy density and pressure are given by

$\rho_{\phi}=\frac{1}{2} \dot{\phi}^{2}+V(\phi), \quad p_{\phi}=\frac{1}{2} \dot{\phi}^{2}-V(\phi)$

where $V(\varphi)$ denotes the potential of the scalar field. In this paper, we consider the interactions between dark matter and dark energy as the following evolution equation

$\rho_{d m}+3 H \rho_{d m}=-\beta \rho_{d m} \dot{\phi}$

where $\beta$ stands for the coupling constant between dark matter and dark energy. The neutrinos are understood to be massless and relativistic particles in the past, but with the coupling to the dark energy, they have acquired mass and became nonrelativistic, having an oscillating mass behavior at low redshifts. In this paper we follow the idea that proposed by [167] and extended by [168]. In the cosmological context, neutrinos cannot be described as fluid. Instead, one must solve the distribution function $f\left(x^{i}, p^{i}, \tau\right)$ in phase space (where $\tau$ is the conformal time). Considering the case that neutrinos are collisionless, the distribution function $f$ does not depend explicitly on time. Solving the Boltzmann equation, one can then calculate the energy density stored in neutrinos ( $f_{0}$ is the background neutrino distribution function):

$$
\begin{aligned}
& \rho_{\nu}=a^{-4} \int q^{2} \sqrt{q^{2}+m_{\nu}(\phi)^{2} a^{2}} f_{0}(q) d q d \Omega \\
& p_{\nu}=\frac{1}{3} a^{-4} \int \frac{q^{2}}{\sqrt{q^{2}+m_{v}(\phi)^{2} a^{2}}} f_{0}(q) d q d \Omega
\end{aligned}
$$

The evolution equation for its energy density according to [168]

$\dot{\rho_{v}}+3 H\left(\rho_{v}+p_{v}\right)=\alpha \dot{\varphi}\left(\rho_{v}-3 p_{v}\right)$

where $\alpha$ denotes coupling constant which can be related neutrino mass $m_{v}$ with relation $\alpha=\frac{d \ln m_{v}}{d \varphi}$. Furthermore, 
from the resulting equations it is possible to obtain evolution equations for the scalar field as

$$
\ddot{\phi}+3 H \dot{\phi}+\frac{d V}{d \phi}+\alpha \dot{\phi}\left(\rho_{v}-3 p_{v}\right)-\beta \rho_{d m} \dot{\phi}=0
$$

For most of the Universe's history, the neutrinos are highly relativistic and $\left(\rho_{v}-3 p_{v}\right) \approx 0$ such that the scalar field and the neutrinos are effectively uncoupled, here only the coupling parameter $\beta$ is important. After the neutrinos become non-relativistic hence the coupling parameter $\alpha$ also becomes important. We consider an exponential potential $V=M_{p l}^{4} \exp \left(-\lambda \frac{\phi}{M_{p l}}\right)$, where $\lambda$ is a dimensionless parameter that determines the slope of the potential. The motivation for choosing these functions have been investigated in [169]. Also we define $\omega=\frac{P_{v}}{\rho_{v}}$. In order to simplify the field equations, we introduce following new variables,

$$
\begin{aligned}
& x_{1}=\frac{\rho_{b}}{3 H^{2}}, x_{2}=\frac{\rho_{v}}{3 H^{2}}, x_{3}=\frac{\rho_{r}}{3 H^{2}}, \\
& x_{4}=\frac{\rho_{d m}}{3 H^{2}}, x_{5}=\frac{\dot{\phi}}{\sqrt{6} H}, x_{6}=\frac{V(\phi)}{3 H^{2}}
\end{aligned}
$$

Hence, the equations of the autonomous dynamical system can be derived as,

$$
\begin{aligned}
& \frac{d x_{1}}{d N}=-3 x_{1}-2 \frac{\dot{H}}{H^{2}} x_{1} \\
& \frac{d x_{2}}{d N}=-x_{2}\left(3(1+\omega)-\sqrt{6} x_{5} \alpha(1-3 \omega)\right)-2 \frac{\dot{H}}{H} x_{2} \\
& \frac{d x_{3}}{d N}=-4 x_{3}-2 \frac{\dot{H}}{H} x_{3} \\
& \frac{d x_{4}}{d N}=-x_{4}\left(3+\sqrt{6} x_{5} \beta+2 \frac{\dot{H}}{H^{2}}\right) \\
& \frac{d x_{5}}{d N}=\frac{3 \lambda}{\sqrt{6}} x_{6}-\frac{3 \alpha(1-3 \omega)}{\sqrt{6}} x_{2}+\frac{9 \beta}{\sqrt{6}} x_{4}-\left(3+\frac{\dot{H}}{H^{2}}\right) x_{5} \\
& \frac{d x_{6}}{d N}=-\sqrt{6} \lambda x_{6} x_{5}-2 \frac{\dot{H}}{H^{2}} x_{6}
\end{aligned}
$$

Where, $N=\ln a$. In term of the new dynamical variable, we also have,

$$
\frac{\dot{H}}{H^{2}}=\frac{1}{2}\left(-3-x_{3}-3 \omega x_{2}-3 x_{5}^{2}+3 x_{6}\right)
$$

In term of new variable the Friedmann equation (1) puts a constraint on new variables as

$$
x_{1}+x_{2}+x_{3}+x_{4}+x_{5}^{2}+x_{6}=1
$$

We demonstrate that for the flat Friedmann-RobertsonWalker model the dynamics can be reduced to the form of the six dimensional autonomous dynamical system where by exerting the constraint (14) it reduces to five dimensional dynamical system. The parameters $\alpha, \beta, \lambda, \omega$ are the free parameters of the model. While the main advantage of the dynamical system methods is that without knowledge of an exact solution it is possible to investigate the properties of the solutions as well as their stability, But this method is not necessarily to check the stability of the system. Rather, even if our goal is to solve equations numerically, this method is a useful method. Because in the solution of the original equations (Friedman and field equations), we are faced with differential equations of order 2 and higher, which are relatively more complex where not only the initial conditions but also the first and second order initial conditions must be determined for any dynamical variable. For example, because of advent of the $\ddot{\varphi}$ in field equation, we need $\varphi(0), \dot{\varphi}(0)$ and $\ddot{\varphi}(0)$ and due to $\dot{H}$ we need $(a(0), H(0)$ and $\dot{H}(0)$ for numerical solutions, however, when the equations are introduced in terms of a set of the first order equations, only the initial condition for the new variable need to be determined $x_{1}(0) \ldots x_{5}(0)$, this makes numerical solution of the equations easier. On the other hand the variables in the dynamical system usually are dimensional lees and in most cases, or at list in the case of Friedman equation we have Pre information about the range of initial conditions. For example, we get $x_{4}=\frac{\rho_{d m}}{3 H^{2}}$ which is $\Omega_{m}$. Hence in numerical solution, it is not necessary to cover a large area of $\Omega_{m}(0)$ but we focus on a small area, approximately between 0.2 and 0.4 . It makes the analysis easier. The importance of this issue becomes more reveal in observational cosmology where the initial condition play important role in the evolution of the universe

However, the question that may arise is that why we don't implement this method for any set of high order differential equations. The answer is that although any second-order differential equation is equivalent to two first-order equations, however the suitable choice of new variables and converting equations to the first order may not be easy except in special cases, but if we could do that then we are faced with a set of first order equations which are more easer to solve.

\section{Observational data, analysis and results}

In what follows, first, we briefly describe the observational data sets used to constrain the parameters of the models under consideration.

- Pantheon: The use of type Ia supernovae ( $\mathrm{SNe}$ ) as standard candles has been of critical importance to cosmology, leading to the discovery of cosmic acceleration $[54,178]$. In this paper, we use the new "Pantheon" sample of Scolnic et al. [179], which adds 276 supernovae from the Pan-STARRS1 Medium Deep Survey at $0.03<z<0.65$ and various low-redshift and HST 
samples to give a total of 1048 supernovae spanning the redshift range $0.01<z<2.3$

The luminosity distance $d_{L}$ can be calculated by

$d_{L}=(1+z) \int \frac{d z}{H(z)}$

In order to incorporate the Eq. (15) with the dynamical system equations of (12), it can be rewriten in terms of the following differential equations

$$
\begin{aligned}
& \frac{d d_{L}}{d N}=-d_{L}-\frac{e^{-2 N}}{H} \\
& \frac{d H}{d N}=H\left(\frac{\dot{H}}{H^{2}}\right)
\end{aligned}
$$

Where, since $1+z \equiv \frac{1}{a}$, then $(1+z) \equiv e^{-N}, d z \equiv$ $-e^{-N} d N$ and $d N \equiv H d t$. Hence by defining new variables $x_{d}=d_{L}$ and $x_{h}=H$ we can write the Eq. (16) as

$$
\begin{aligned}
& \frac{d x_{d}}{d N}=-x_{d}-\frac{e^{-2 N}}{x_{h}} \\
& \frac{d x_{h}}{d N}=-x_{h}\left(\frac{\dot{H}}{H^{2}}\right)
\end{aligned}
$$

The Eq. (18) are related with Eq. (12) by $\frac{\dot{H}}{H^{2}}$ which have been obtained in terms on new variables. It is also important to note that $\frac{\dot{H}}{H^{2}}$ play the important role in cosmology, since important cosmological parameters such as deceleration parameters $q$ an effective equation of state (EoS) $w_{\text {eff }}$ can be expressed in terms of this parameter as $q=-1-\frac{\dot{H}}{H^{2}}$ and $w_{\text {eff }}=-1-\frac{2}{3} \frac{\dot{H}}{H^{2}}$. Hence in order to find $x_{d}$ and $x_{h}$ the set of Eqs. (18) and (12) must be coupled and solved simultaneously. Hence the distance modulus also can be obtained as $\mu_{t h}(z)=5 \log \left(x_{d}\right)+42.38$. we can compute the $\chi^{2}$-statistics for each case. Therefore, we proceed to define the following quantities:

$\chi_{\text {Pantheon }}^{2}=\sum_{i}^{N_{\text {Pantheon }}} \frac{\left(\mu\left(z_{i}\right)_{\mathrm{obs}}-\mu\left(z_{i}\right)_{\mathrm{th}}\right)^{2}}{\sigma\left(z_{i}\right)_{\mathrm{obs}, \text { Pantheon }}^{2}}$,

where $N$ is the number of data points, $\sigma_{i}$ is the uncertainty associated with each measurement.

- Cosmic Microwave Background(CMB): The observations of temperature anisotropies in the CMB provide a valuable independent test for the reality of dark energy at the recombination epoch $z$ 1090. The photons were coupled to baryons and electrons before that red shift and decoupled right after. Due to the fact that in the Boltzmann and Einstein equations all the components of the universe are coupled, in order to extract information from the full spectrum, demanding numerical simulations are needed. A convenient and efficient way to summarize information from the $\mathrm{CMB}$ data, without using the full spectrum, is by employing the so called CMB shift parameters or distance priors.

The CMB shift parameter $R$, given by $[180,181]$

$R=\Omega_{m 0}^{\frac{1}{2}} \int_{0}^{z_{r e c}} \frac{d z}{E(z)}$

where $E(z)=\frac{H(z)}{H_{0}}$ and $z_{\text {rec }}$ is the redshift of recombination $z_{r e c}=1090$ [182]. The parameter $R$ ties up the angular diameter distance to the last scattering surface, the comoving size of the sound horizon at $z=1091.3$ and the angular scale of the first acoustic peak in CMB power spectrum of temperature fluctuations $[180,181]$. The updated value of $\mathrm{R}$ from WMAP5 is $R_{o b s}=1.710 \pm 0.019$ [183]. The $\chi_{C M B}^{2}$ for the CMB data is

$\chi_{C M B}^{2}=\frac{\left(R-R_{o b s}\right)^{2}}{\sigma_{R}}$

where the corresponding $1 \sigma$ errors is $\sigma_{R}=0.019$.

\section{- Baryon acoustic oscillations BAO data}

For BAO data, from the measurement of the BAO peak in the distribution of SDSS luminous red galaxies, we define parameter A as [184]

$A=\Omega_{m 0}^{\frac{1}{2}} E\left(z_{b}\right)^{\frac{-1}{3}}\left[\frac{1}{z_{b}} \int_{0}^{z_{b}} \frac{d z}{E(z)}\right]^{\frac{2}{3}}$

where $z_{b}=0.35$. The SDSS BAO measurement [184] gives $A_{o b s}=0.469\left(n_{s} / 0.98\right) .0 .35 \pm 0.017$, where the scalar spectral index is taken to be $n_{s}=0.965$ as measured by Planck 2018 [185]. The parameter A is nearly model-independent and imposes the robust constraint as complement to SNIa data. The $\chi^{2}$ for the BAO data is

$\chi_{B A O}^{2}=\frac{\left(A-A_{o b s}\right)^{2}}{\sigma_{A}}$

where the corresponding $1 \sigma$ errors is $\sigma_{A}=0.017$. Table 1 shows the best fitted model parameters and initial conditions in both power law and exponential cases.

- CC We use the cosmic chronometers (CC) data set comprising of 36 measurements spanning the redshift range 
$z \leq 2.36$, recently compiled in [186]

$\chi_{\mathrm{CC}}^{2}=\sum_{i}^{N_{\mathrm{CC}}} \frac{\left(H\left(z_{i}\right)_{\mathrm{obs}}-H\left(z_{i}\right)_{\mathrm{th}}\right)^{2}}{\sigma\left(z_{i}\right)_{\mathrm{obs}, \mathrm{CC}}^{2}}$,

In order to put constraints on the parameters of the model we must note that the model has five independent variables $\left(x_{1}, x_{2}, x_{3}, x_{4}, x_{5}\right)$ which according to 11 are equivalent to $\left(\Omega_{b}, \Omega_{v}, \Omega_{r}, \Omega_{d m}, \Omega_{\dot{\phi}}^{1 / 2}\right)$ as well as four free parameters of the model $(\alpha, \beta, \lambda, \omega)$. Hence in order to solve the equation numerically the five initial conditions $\left(x_{1}(0), . ., x_{5}(0)\right)$ and value of the parameters must be known. In observational measurements one or more parameter are added to the free parameters. For example in numerical analysis using Pantheon data the two new variables $x_{d}=d_{L}$ and $x_{h}=H$ and for CC, CMB and BAO data the variable $x_{h}=H$ are added to the free parameters. The other parameters are expressed in terms of the main parameters and can be constrained indirectly. For example $\sum m_{v}$ can be related to the main parameters $\left(h, \Omega_{v}\right)$ as

$\Omega_{v}=\frac{\sum m_{v}}{94 h^{2} \mathrm{eV}}$

where $h$ is the reduced Hubble constant (the Hubble constant $H_{0}=100 h \mathrm{~km} / \mathrm{s} / \mathrm{Mpc}$. Hence if the parameters $\left(h, \Omega_{v}\right)$ are constrained then the parameter $\sum m_{v}$ is constrained automatically.

The relativistic energy density in the early universe include the contributions from photons and neutrinos, and possibly other extra relativistic degrees of freedom, called dark radiation. The effective number of relativistic species, including neutrinos and any other dark radiation, is defined by a parameter, $N_{e f f}$, for which the standard value is 3.046 corresponding to the case with threegeneration neutrinos and no extra dark radiation [187]. If the value of $N_{\text {eff }}$ is beyond 3.046, it indicates that there is some dark radiation other than three-generation active neutrinos. The behaviour of dark radiation is exactly equivalent to massless neutrinos. Thus, the total radiation energy density in the Universe is given by

$\rho_{r}=\rho_{\gamma}\left[1+\frac{7}{8}\left(\frac{4}{11}\right)^{\frac{4}{3}} N_{e f f}\right]$

where $\rho_{\gamma}$ is the energy density of photons. We parametrize the relativistic degrees of freedom using the effective number of neutrino species, $N_{e f f}$. This quantity can be written in terms of the matter density, $\Omega_{m} h^{2}$, and the redshift of matter-radiation equality $z_{e q}$ as [182]

$$
N_{e f f}=3.04+7.44\left(\frac{\Omega_{m} h^{2}}{0.1308} \frac{3139}{1+z_{e q}}-1\right)
$$

\section{Results}

Throughout this section we will present the results obtained within the two different IDE scenario

\subsection{IDE $+\sum m_{v}$}

The results for the cosmological parameters within this interacting dark energy model are shown in Table 1. Figure 1 also show the parametric space at $68 \% \mathrm{CL}$ and $95 \% \mathrm{CL}$ for some selected parameters for the different observational data sets. In order to compare our results with those obtained by Planck 2018 [185], we have listed some of the Planck 2018 results [185] in Table 2. For this case the free parameters are $\left(\sum m_{v}, h, \Omega_{m}, \Omega_{b} h^{2}, \Omega_{c} h^{2}, \alpha, \beta, \lambda, \omega\right)$.

From the analyses of the Pantheon data alone, as shown in Table 1, we find that

$\sum m_{v}<0.253 \mathrm{eV} 95 \% C L$

This result is very close to the result of [121], the case IDE+ $\sum m_{v}$, using $\left(\mathrm{CMB}+\right.$ Pantheon $+\mathrm{CC}$ data) with $\sum m_{v}<$ $0.255 \mathrm{eV}$ at $95 \% \mathrm{CL}$. Both the model is similar to ours and the data used includes Pantheon data. The result is also closed to the result of [122], the case interacting vacuum scenario (IVS) $+\sum m_{v}$, with $\sum m_{v}<0.277 \mathrm{eV}$ at $95 \% \mathrm{CL}$ ) and the result of Planck 2018 [185] the case (TT, TE, EE + lowE + lensing) with $\sum m_{v}<0.241 \mathrm{eV}$ at $95 \% \mathrm{CL}$. Using CC data, we find that

$\sum m_{v}<0.199 \mathrm{eV} 95 \% C L$

which is close to the result of [121], the case IDE $+\sum m_{v}$ using $\left(\mathrm{CMB}+\right.$ Pantheon+CC data) with $\sum m_{v}<0.159 \mathrm{eV}$ at $95 \%$ CL and the result of Planck 2018 [185] the case (TT, lowE + BAO) with $\sum m_{v}<0.16 \mathrm{eV}$ at $95 \% \mathrm{CL}$.

Using $\mathrm{CMB}+\mathrm{BAO}$, we find that

$\sum m_{v}<0.33 \mathrm{eV} 95 \% C L \quad C M B+B A O$

This result is very close to the result of [121], the case IDE+ $\sum m_{v}$, using (CMB data) with $\sum m_{v}<0.313 \mathrm{eV} 95 \% \mathrm{CL}$ and comparable with and comparable with results of [185] the case TT, TE, EE + lowE[CamSpec] with $\sum m_{v}<0.38$ $\mathrm{eV}$ 95\% CL 

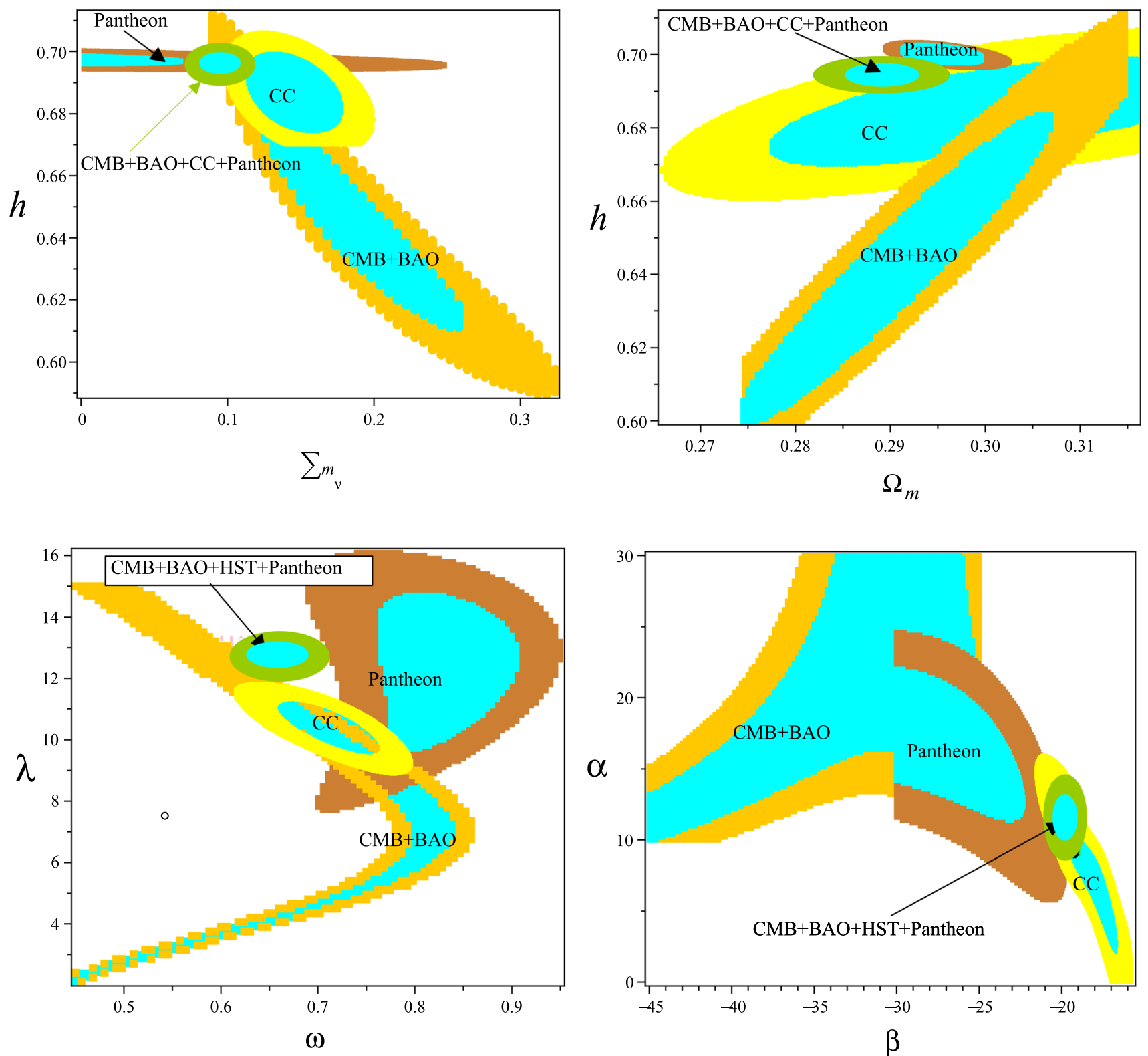

Fig. 1 The constraints at the $68 \%$ and $95 \%$ CL two-dimensional contours for selected cosmological parameters and parameters of the model parameters in IDE $+\sum m_{v}$ scenario for the Pantheon, $\mathrm{CC}, \mathrm{CMB}+\mathrm{BAO}$ and Pantheon $+\mathrm{CC}+\mathrm{CMB}+\mathrm{B} \mathrm{AO}$ dataset

For combination of full data, Pantheon $+\mathrm{CC}+\mathrm{CMB}+$ BAO, we find

$\sum m_{v}<0.125 \mathrm{eV} 95 \% C L$

The result is very close to results of [185] TT, TE, EE + lowE + lensing + BAO with $\sum m_{v}<0.12 \mathrm{eV}$ at $95 \% \mathrm{CL}$ and case TT, TE, EE + lowE + BAO with $\sum m_{v}<0.13 \mathrm{eV}$ at $95 \% \mathrm{CL}$. It is also comparable with that obtained by [121] with $\sum m_{v}<0.156 \mathrm{eV}$ at $95 \% \mathrm{CL}$ using same model IDE+ $\sum m_{v}$ and same dada (Pantheon $\left.+\mathrm{CC}+\mathrm{CMB}+\mathrm{BAO}\right)$

We also put constraint on coupling parameters $(\alpha, \beta, \lambda, \omega)$. The lower panel of Fig. 1 shows $(68.3 \%, 95 . \%)$ confidence levels for the parameters $(\alpha, \beta)$ and $(\lambda, \omega)$ for Pantheon, $\mathrm{CC}$ and $\mathrm{CMB}+\mathrm{BAO}$ and combination of the data Pantheon $+\mathrm{CC}+\mathrm{CMB}+\mathrm{BAO}$. The results also have been listed in Table 1. Constraining on parameter $\lambda$, we have obtained $\lambda 11.85_{-4.35}^{+4.35}, \lambda=10.35_{-1.55}^{+1.55}, \lambda=8.6_{-6.70}^{+6.70}$ and $\lambda=12.8_{-0.90}^{+0.90}$ at $68 \% \mathrm{CL}$ for Pantheon, $\mathrm{CC}, \mathrm{CMB}+\mathrm{BAO}$ and Pantheon $+\mathrm{CC}+\mathrm{CMB}+\mathrm{BAO}$ respectively. Since we have considered $V(\phi)=m_{p l}^{4} \exp \left(\frac{-\lambda \phi}{m_{p l}}\right)$, the positive value of $\lambda$ indicates that potential $V(\varphi)$ is a monotonically decreasing function of $\varphi$. and has a negative gradient. It is also important to note that the best fitted values of $\lambda$ for both individual and combination data values are very close to that deter- 

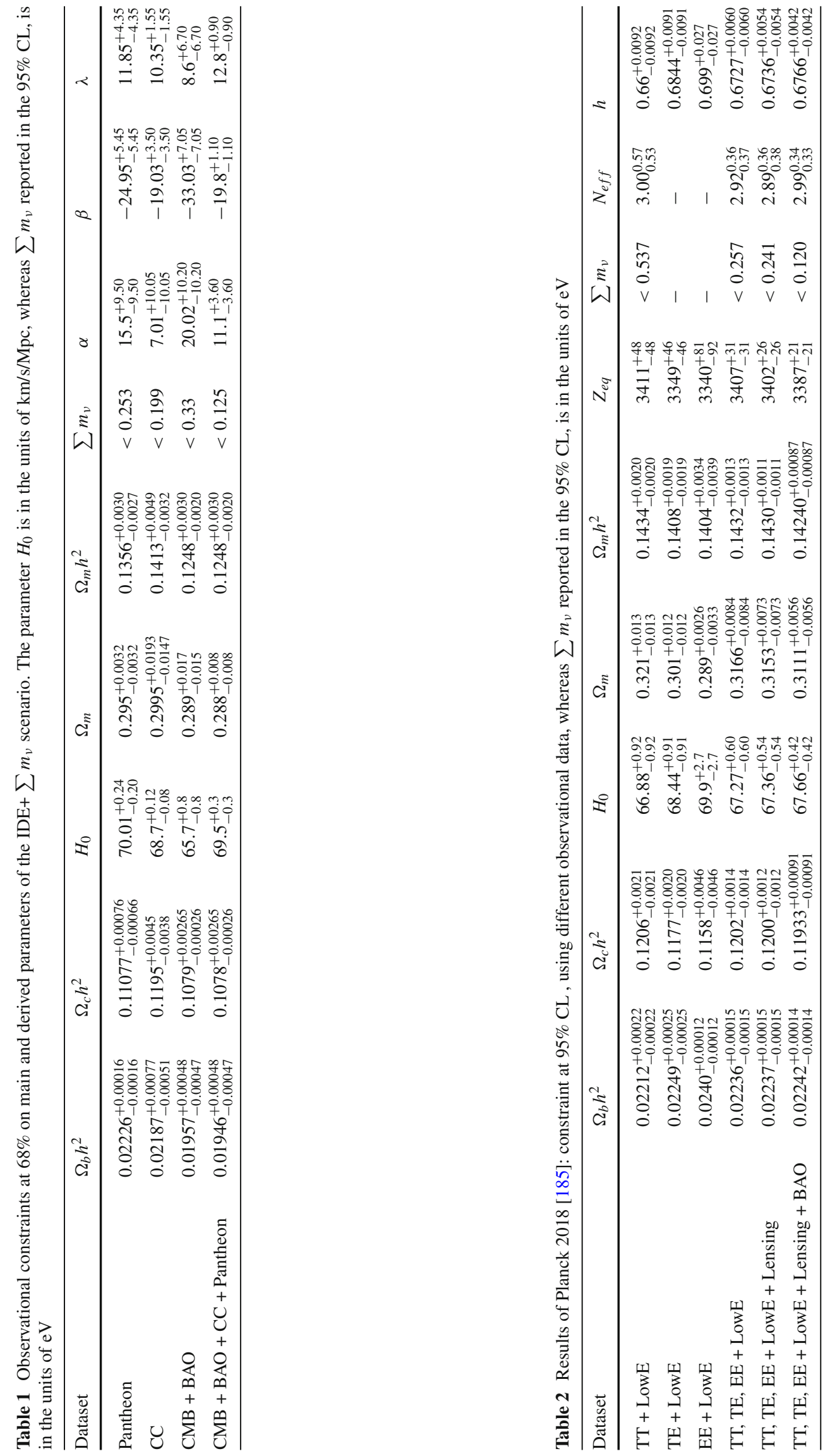
mined by upper bounds on early dark energy $(\lambda \geq 10)[161]$. In fact, when the potential energy approaches a constant $V(\phi)=m_{p l}^{4} \exp \left(\frac{-\lambda \phi}{m_{p l}}\right) \rightarrow V_{t}=V(\phi)=m_{p l}^{4} \exp \left(\frac{-\lambda \phi_{t}}{m_{p l}}\right)$, where the evolution of the cosmon field stops close to a value $\phi_{t}$ which is characteristic for the transition between the two different cosmological epochs, it acts similar to a cosmological constant and causes the accelerated expansion and for $\frac{\lambda \phi_{t}}{m_{p l}} \simeq 276$ the cosmological constant has a value compatible with observation. This amount gives $\lambda \geq 10$ (for more discussion see [161])

For most of the Universe's history, the neutrinos are highly relativistic and $\left(\rho_{v}-3 p_{v}\right) \approx 0$ such that the scalar field and the neutrinos are effectively uncoupled, here only the coupling parameter $\beta$ is important. After the neutrinos become non-relativistic hence the coupling parameter $\alpha$ also becomes important. Constraining on parameter $\alpha$, we have obtained $\alpha=15.5_{-9.50}^{+9.50}, \alpha=7.01_{-10.05}^{+10.05}, \alpha=20.02_{-10.20}^{+10.20}$ and $\lambda=11.1_{-3.60}^{+3.60}$ at $68 \% \mathrm{CL}$ for Pantheon, CC, CMB + BAO and Pantheon $+\mathrm{CC}+\mathrm{CMB}+\mathrm{BAO}$ respectively. As investigated by [188], the following conditions must be met to give rise to growing neutrino quintessence:

- $V(\varphi)$ must have a negative gradient in order to cause the value of the scalar field to increase with time. This gradient must be sufficiently steep that $\varphi$ reaches large enough values in the late Universe to act as dark energy.

- $|\alpha|$ must be sufficiently large when the neutrinos become non-relativistic that $\beta\left(\rho_{v}-3 p_{v}\right)$ is able to act as a strong enough restoring force to stop the evolution of $\varphi$ in Eq. (10).

The best fitted of $(\alpha, \lambda)$ satisfy the above condition. Despite the small value of $\Omega_{\nu}$ the neutrinos are important for the evolution of the cosmon due to their large coupling $\alpha$

For both individual and combination of the dataset, we find that the large value for coupling parameter $\beta$. We find $|\beta|>19 \mid$ at $(95 \% \mathrm{CL}$. This indicates there is a strong interaction between dark matter and dark energy.

\subsection{IDE $+\sum m_{v}+N_{e f f}$}

The results for the cosmological parameters within this interacting dark energy model are shown in Table 3. Figure 2 Also show the parametric space at $68 \% \mathrm{CL}$ and 95\% CL for some selected parameters for the different observational data sets. For this case the free parameters are $\left(\sum m_{v}, N_{e f f}, h, \Omega_{m}, \Omega_{b} h^{2}, \Omega_{c} h^{2}, \alpha, \beta, \lambda, \omega\right)$.

Although the results for this case, IDE $+\sum m_{v}+N_{\text {eff }}$, are consistent with those in the previous case IDE $+\sum m_{v}$ and the presence of $N_{e f f}$ does not significantly shift the result, 

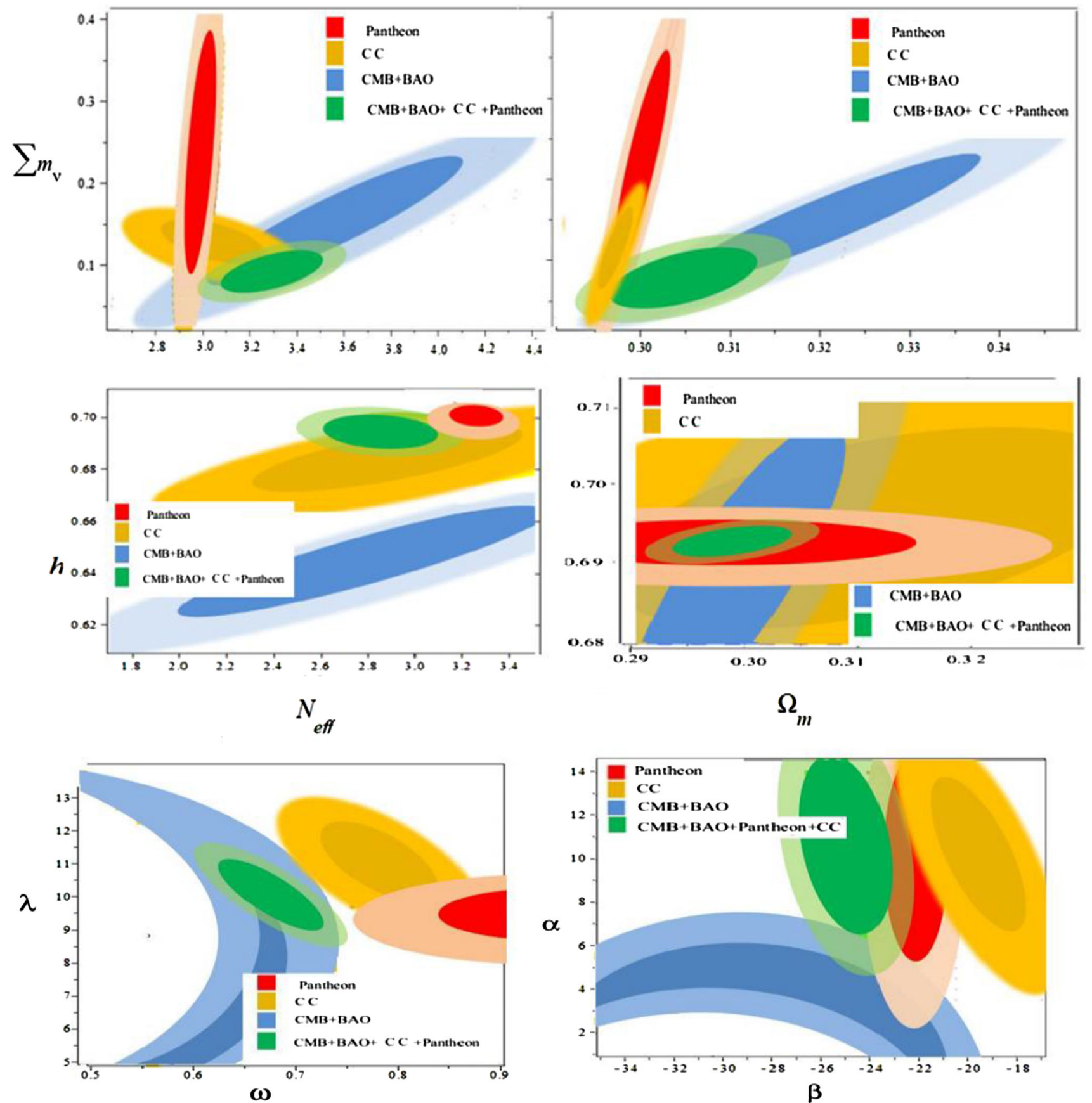

Fig. 2 The constraints at the $68 \%$ and $95 \% \mathrm{CL}$ two-dimensional contours for selected cosmological parameters and parameters of the model in IDE $+\sum m_{v}+N_{e f f}$ scenario for the Pantheon, CC, CMB + BAO and Pantheon $+\mathrm{CC}+\mathrm{CMB}+\mathrm{BAO}$ dataset

however the bounds on some of the parameters have been changed slightly.

From the analyses of the Pantheon data alone, as shown in Table 3, we find that

$\sum m_{v}<0.45 \mathrm{eV} 95 \% C L$
Which in comparison of previous model, IDE $+\sum m_{\nu}$, with $\sum m_{v}<0.253 \mathrm{eV}$ have been changed significantly, however it is close to the result of [189] where using same model, interacting scenario IDE1 $1 \mathrm{p}+\sum m_{v}+N_{\text {eff }}$ and using Planck 2018 data, have been obtained $\sum m_{v}<0.438 \mathrm{eV}$ at $95 \% \mathrm{CL}$ The interesting result of our analysis is that for this model, the most stringent upper limit we have on this parameter is obtained for the data set combination $C M B+B A O+$ 
Pantheon $+C C$ in which

$\sum m_{v}<0.125 \mathrm{eV} 95 \% C L$

This result is in good agreement with the results of Planck 2018 [185], where the limit of the total neutrino mass is $\sum m_{v}<0.12 \mathrm{eV}$ at $(95 \%$ C.L. using TT, TE, EE + lowE + lensing + BAO data) and close to the result of [121], where they also find the most stringent upper limit on this parameter for the same model, IDE $+\sum m_{v}$ and the same data $(\mathrm{CMB}+$ Pantheon + CC data) with $\sum m_{v}<0.15 \mathrm{eV}$ at $95 \% \mathrm{CL}$. For combination data we also find

$N_{e f f}=2.955_{-0.12}^{+0.11} 68 \% C L$

which is very close to the result of Planck 2018 [185] with $N_{\text {eff }}=2.96_{-0.33}^{+0.34}$ at $68 \% \mathrm{CL}$, the case TT,TE,EE,LowE + lensing+BAO and close to result of [121] with $N_{e f f}=$ $3.02_{-0.33}^{+0.34}$ using the same data and same model.

For this case, the best fitted values for $\lambda$, have obtained as $\lambda=9.7_{-0.8}^{+0.8}, \lambda=11.1_{-1.05}^{+1.05}, \lambda=7.9_{-3.3}^{+3.3}$ and $\lambda=10.25_{-1.08}^{+1.05}$ at $68 \% \mathrm{CL}$ for Pantheon, CC, CMB + BAO respectively. Also for combination of dataset, $\mathrm{CMB}+$ Pantheon $+\mathrm{CC}$ data we have found

$\lambda=10.25_{-1.08}^{+1.05}$

The results are close to those obtained in previous case, IDE+ $\sum m_{v}$. Hence same as the previous case, the positive values of $\lambda$ indicate that potential $V(\varphi)$ is a monotonically decreasing function of $\varphi$. and has a negative gradient as well as these value are consistence with that predicted by observation.

\section{Conclusion}

In this paper, we have explored possible extensions of the Interacting Dark Energy, where the dark energy and the dark matter fluids interact with each other.

We have considered a cosmological model in which dark energy and neutrinos are coupled such that the mass of the neutrinos and potentials are function of the scalar field as $m_{v}=m_{0} \exp \left(\frac{\alpha \phi}{m_{p l}}\right)$ and $V(\phi)=m_{p l}^{4} \exp \left(\frac{\lambda \phi}{m_{p l}}\right)$ respectively. While the theoretical aspects of the neutrino-dark energy interaction have been studied in previous studies [167-169,188], here we have extended the model such that not only neutrino but also dark matter interact with dark energy with different coupling terms $Q_{d e-d m}=\frac{\beta}{m_{p}} \dot{\phi} \rho_{d m}$ and $Q_{d e-v}=\frac{\alpha}{m_{p}}\left(\rho_{v}-3 p_{v}\right) \dot{\phi}$ and focused on observational aspect of the model. We have exploited the most recent publicly available cosmological observations, which include the Supernovae Type Ia Pantheon data and measurements of the Hubble parameter from Cosmic Chronometers. CMB data,
Baryon Acoustic Oscillations data (BAO) to put constrain on parameters on $\sum m_{v}, N_{e f f}, z_{e q}, \Omega_{m}, \Omega_{c}, \Omega_{b}, \Omega_{h}, \alpha, \beta, \lambda, \omega$ We find that while the results of individuals and combination of datasets are closed, the most stringent upper limit we have on this parameter is obtained for the data set combination $C M B+B A O+$ Pantheon $+C C$ in which $\sum m_{v}<$ $0.125 \mathrm{eV} 95 \% C L$ This result is in good agreement with the results of Planck 2018 [185], where the limit of the total neutrino mass is $\sum m_{v}<0.12 \mathrm{eV}$ at $(95 \%$ C.L. using TT,TE,EE+lowE+lensing+BAO data) and close to the result of [121], where they also find the most stringent upper limit on this parameter ..for the same model, IDE $+\sum m_{v}$ and same data $\left(\mathrm{CMB}+\right.$ Pantheon + CC data) with $\sum m_{v}<0.15 \mathrm{eV}$ at 95\% CL.

This study also investigated one of the main problem in growing neutrinos and cosmological selection. As point out by Amendola and Wetterich [190], the most crucial observational issues can be understood by understanding on constant parameters $(\lambda, \alpha, \beta)$ it will be a challenge to measure them or to falsify the growing matter scenario. For neutrino growing matter a determination of $\lambda$ and $\alpha$ would fix the neutrino mass, allowing for an independent test of this hypothesis by comparing with laboratory experiments. The values obtained for these parameters in this study are close to those require for growing matter mechanism.

For both model IDE $+\sum m_{v}$ and IDE $+\sum m_{v}+N_{e f f}$ and for all dataset, Both individual and combination, we find the mean value of $\lambda$ as $\lambda \simeq 10$. This value is very close to that determined by upper bounds on early dark energy $(\lambda \geq 10)$ [161]. As also point out by $V(\varphi)$ must have a negative gradient in order to cause the value of the scalar field to increase with time. This gradient must be sufficiently steep that $\varphi$ reaches large enough values in the late Universe to act as dark energy. Since we get $V(\phi)=m_{p l}^{4} \exp \left(\frac{-\lambda \phi}{m_{p l}}\right)$, the best fitted values of $\lambda$ which is positive satisfies this condition.

Data Availability Statement This manuscript has no associated data or the data will not be deposited. [Authors' comment: The paper has no external data.]

Open Access This article is licensed under a Creative Commons Attribution 4.0 International License, which permits use, sharing, adaptation, distribution and reproduction in any medium or format, as long as you give appropriate credit to the original author(s) and the source, provide a link to the Creative Commons licence, and indicate if changes were made. The images or other third party material in this article are included in the article's Creative Commons licence, unless indicated otherwise in a credit line to the material. If material is not included in the article's Creative Commons licence and your intended use is not permitted by statutory regulation or exceeds the permitted use, you will need to obtain permission directly from the copyright holder. To view a copy of this licence, visit http://creativecomm ons.org/licenses/by/4.0/.

Funded by $\mathrm{SCOAP}^{3}$. 


\section{References}

1. A.G. Reiss et al., Astron. J. 116, 1009 (1998)

2. C.I. Bennet et al., Astrophys. J. Suppl. 148, 1 (2003)

3. A.G. Riess, et al., [Supernova Search TeamCollaboration] Astron. J. 116, 1009 (1998)

4. A.C. Pope et al., Astrophys. J. 607, 655 (2004)

5. D.N. Spergel et al., Astrophys J. Suppl. 148, 175 (2003)

6. V. Sahni, A. Starobinsky, Int. J. Mod. Phys. D 9, 373-444 (2000)

7. S. Weinberg, Rev. Mod. Phys. 61, 1 (1989)

8. J.P. Ostriker, P.J. Steinhardt, arXiv:astro-ph/9505066

9. L. Verde, T. Treu, A.G. Riess (2019) arXiv:1907.10625 [astroph.CO]

10. R.R. Caldwell, R. Dave, P.J. Steinhardt, Phys. Rev. Lett. 80, 1582 (1998)

11. I. Zlatev, L. Wang, P.J. Steinhardt, Phys. Rev. Lett. 82, 896 (1999)

12. B. Wang, E. Abdalla, F. Atrio-Barandela, D. Pavon, Rep. Prog. Phys. 79, 096901 (2016). arXiv:1603.08299 [astro-ph.CO]

13. E. Di Valentino, A. Melchiorri, O. Mena, S. Vagnozzi, Phys. Rev. D 101, 063502 (2020)

14. E. Di Valentino, A. Melchiorri, O. Mena, S. Vagnozzi, Phys. Dark Univ. 30, 100666 (2020)

15. S. Pan, W. Yang, E. Di Valentino, E.N. Saridakis, S. Chakraborty, Phys. Rev. D 100, 103520 (2020)

16. W. Yang, S. Pan, E. Di Valentino, R.C. Nunes, S. Vagnozzi, D.F. Mota, JCAP 1809, 019 (2018)

17. W. Yang, S. Pan, E. Di Valentino, O. Mena, A. Melchiorri

18. R.R. Caldwell, M. Kamionkowski, N.N. Weinberg, Phys. Rev. Lett. 91, 071301 (2003)

19. Z.H. Zhu, M.K. Fujimoto, X.T. He, Astrophys. J. 603, 365-370 (2004)

20. Z.H. Zhu, J.S. Alcaniz, Astrophys. J. 620, 7-11 (2005)

21. J. Sadeghi, M.R. Setare, A. Banijamali, F. Milani, Phys. Lett. B 662, 92 (2008)

22. J. Sadeghi, M.R. Setare, A. Banijamali, F. Milani, Phy. Rev. D 79, 123003 (2009)

23. Z.K. Guo et al., Phys. Lett. B 608, 177 (2005)

24. J.-Q. Xia, B. Feng, X. Zhang, Mod. Phys. Lett. A 20, 2409 (2005)

25. M.R. Setare, Phys. Lett. B 641, 130 (2006)

26. W. Zhao, Y. Zhang, Phy. Rev. D 73, 123509 (2006)

27. G.-B. Zhao, J.-Q. Xia, B. Feng, X. Zhang, Int. J. Mod. Phys. D 16, 1229 (2007)

28. M.R. Setare, J. Sadeghi, A.R. Amani, Phys. Lett. B 660, 299 (2008)

29. M.R. Setare, E.N. Saridakis, Phys. Lett. B 668, 177 (2008)

30. M.R. Setare, E.N. Saridakis, Int. J. Mod. Phys. D 18, 549 (2009)

31. M.R. Setare, E.N. Saridakis, J. Cosmol. Astrophys. 09, 026 (2008)

32. Y.F. Cai, T. Qiu, Y.S. Piao, M. Li, X. Zhang, JHEP 0710, 071 (2007)

33. H. Farajollahi, A. Salehi, F. Tayebi, A. Ravanpak, J. Cosmol. Astrophys. 05, 017 (2011)

34. S. Capozziello, V.F. Cardone, S. Carloni, A. Troisi, Int. J. Mod. Phys. D 15, 69 (2006)

35. S. Capozziello, V.F. Cardone, S. Carloni, A. Troisi, Int. J. Mod. Phys. D 12, 1969 (2003)

36. M.R. Setare, Phys. Lett. B 644, 99-103 (2007)

37. M.R. Setare, M. Jamil, Phys. Lett. B 690, 1-4 (2010)

38. A.C. Davis, C.A.O. Schelpe, D.J. Shaw, Phy. Rev. D 80, 064016 (2009)

39. Y. Ito, S. Nojiri, Phys. Rev. D 79, 103008 (2009)

40. T. Tamaki, S. Tsujikawa, Phys. Rev. D 78, 084028 (2008)

41. H. Farajollahi, A. Salehi, Int. J. Mod. Phys. D 19, 621-633 (2010b)

42. D.F. Mota, D.J. Shaw, Phy. Rev. D 75, 063501 (2007)

43. K. Dimopoulos, M. Axenides, J. Cosmol. Astrophys. 0506, 008 (2005)
44. T. Damour, G.W. Gibbons, C. Gundlach, Phys. Rev. Lett. 64, 123 (1990)

45. M.R. Setare, E.C. Vagenas, Int. J. Mod. Phys. D 18, 147-157 (2009)

46. S.M. Carroll, Phys. Rev. Lett. 81, 3067 (1998)

47. S.M. Carroll, W.H. Press, E.L. Turner, Ann. Rev. Astron. Astrophys. 30, 499 (1992)

48. T. Biswas, R. Brandenberger, A. Mazumdar, T. Multamaki, Phys. Rev. D 74, 063501 (2006)

49. J.P. Uzan, Rev. Mod. Phys. 75, 403 (2003)

50. B. Bertotti et al., Nature 425, 374 (2003)

51. G.F. Chew, S.C. Frautschi, Phys. Rev. Lett. 7, 394 (1961)

52. T. Damour, F. Piazza, G. Veneziano, Phys. Rev. D 66, 046007 (2002)

53. S. Nojiri, S.D. Odintsov, Mod. Phys. Lett. A 19, 1273-1280 (2004)

54. A.G. Riess, A.V. Filippenko, P. Challis et al., Observational evidence from supernovae for an accelerating universe and a cosmological constant. AJ 116, 1009 (1998). arXiv:astro-ph/9805201

55. Y. Fukuda, T. Hayakawa, E. Ichihara, K. Inoue, K. Ishihara, H. Ishino et al., Measurements of the solar neutrino flux from superKamiokande's first 300 days. Phys. Rev. Lett. 81, 1158-1162 (1998). arXiv:hep-ex/9805021

56. Q.R. Ahmad et al. (SNO Collaboration), Phys. Rev. Lett. 87, 071301(2001)

57. B. Pontecorvo, Mesonium and anti-mesonium. Sov. Phys. JETP 6, 429 (1957)

58. A. Osipowicz et al. [KATRIN Collaboration], KATRIN: a next generation tritium beta decay experiment with sub-eV sensitivity for the electron neutrino mass. Letter of intent, arXiv:hep-ex/0109033

59. H.V. Klapdor-Kleingrothaus, U. Sarkar, Implications of observed neutrinoless double beta decay. Mod. Phys. Lett. A 16, 2469 (2001). https://doi.org/10.1142/S0217732301005850

60. H.V. Klapdor-Kleingrothaus, I.V. Krivosheina, A. Dietz, O. Chkvorets, Search for neutrino- less double beta decay with enriched Ge-76 in Gran Sasso 1990-2003. Phys. Lett. B 586, 198 (2004). https://doi.org/10.1016/j.physletb.2004.02.025. arXiv:hep-ph/0404088

61. C. Kraus et al., Final results from phase II of the Mainz neutrino mass search in tritium beta decay. Eur. Phys. J. C 40, 447 (2005). https://doi.org/10.1140/epjc/s2005-02139-7. arXiv:hep-ex/0412056

62. E.W. Otten, C. Weinheimer, Neutrino mass limit from tritium beta decay. Rep. Prog. Phys. 71, 086201 (2008). https://doi.org/10. 1088/0034-4885/71/8/086201. arXiv:0909.2104 [hep-ex]

63. J. Wolf [KATRIN Collaboration], The KATRIN neutrino mass experiment. Nucl. Instrum. Methods A 623, 442 (2010). https://doi.org/10.1016/j.nima.2010.03.030. arXiv:0810.3281 [physics.ins-det]

64. G.Y. Huang, S. Zhou, Discriminating between thermal and nonthermal cosmic relic neutrinos through an annual modulation at PTOLEMY. Phys. Rev. D 94(11), 116009 (2016). arXiv: 1610.01347

65. J.W.F. Valle, Neutrino masses and oscillations. AIP Conf. Proc. 805(1), 128 (2005). https://doi.org/10.1063/1.2149688. arXiv:hep-ph/0509262

66. S. Hannestad, Neutrino physics from precision cosmology. Prog. Part. Nucl. Phys. 65, 185 (2010). https://doi.org/10.1016/j.ppnp. 2010.07.001. arXiv: 1007.0658 [hep- ph]

67. J. Lesgourgues, S. Pastor, Neutrino mass from cosmology. Adv. High Energy Phys. 2012, 608515 (2012). https://doi.org/10.1155/ 2012/608515. arXiv:1212.6154 [hep-ph]

68. K.N. Abazajian et al. [Topical Conveners: K.N. Abazajian, J.E. Carlstrom, A.T. Lee Collaboration], Neutrino physics from the cosmic microwave back-ground and large scale struc- 
ture. Astropart. Phys. 63, 66 (2015) https://doi.org/10.1016/j. astropartphys.2014.05.014. arXiv:1309.5383 [astro-ph.CO]

69. W. Hu, D.J. Eisenstein, M. Tegmark, Weighing neutrinos with galaxy surveys. Phys. Rev. Lett. 80, 5255 (1998)

70. B.A. Reid, L. Verde, R. Jimenez, O. Mena, Robust neutrino constraints by combining low redshift observations with the CMB. JCAP 1001, 003 (2010)

71. S.A. Thomas, F.B. Abdalla, O. Lahav, Upper bound of $0.28 \mathrm{eV}$ on the neutrino masses from the largest photometric redshift survey. Phys. Rev. Lett. 105, 031304 (2010)

72. C. Carbone, L. Verde, Y. Wang, A. Cimatti, Neutrino constraints from future nearly all-sky spectroscopic galaxy surveys. JCAP 1103, 030 (2011)

73. H. Li, X. Zhang, Constraining dynamical dark energy with a divergence-free parametrization in the presence of spatial curvature and massive neutrinos. Phys. Lett. B 713, 160 (2012)

74. X. Wang, X.L. Meng, T.J. Zhang, H. Shan, Y. Gong, C. Tao, X. Chen, Y.F. Huang, Observational constraints on cosmic neutrinos and dark energy revisited. JCAP 1211, 018 (2012). https://doi. org/10.1088/1475-7516/2012/11/018. arXiv:1210.2136 [astroph.CO]

75. Y.H. Li, S. Wang, X.D. Li, X. Zhang, Holographic dark energy in a universe with spatial curvature and massive neutrinos: a full Markov Chain Monte Carlo exploration. JCAP 1302, 033 (2013)

76. B. Audren, J. Lesgourgues, S. Bird, M.G. Haehnelt, M. Viel, Neutrino masses and cosmological parameters from a Euclid-like survey: Markov Chain Monte Carlo forecasts including theoretical errors. JCAP 1301, 026 (2013)

77. S. Riemer-S $\phi$ rensen, D. Parkinson, T.M. Davis, Combining Planck data with large scale structure information gives a strong neutrino mass constraint. Phys. Rev. D 89, 103505 (2014)

78. A. Font-Ribera, P. McDonald, N. Mostek, B.A. Reid, H.J. Seo, A. Slosar, DESI and other dark energy experiments in the era of neutrino mass measurements. JCAP 1405, 023 (2014)

79. J.F. Zhang, Y.H. Li, X. Zhang, Sterile neutrinos help reconcile the observational results of primordial gravitational waves from Planck and BICEP2. Phys. Lett. B 740, 359 (2015)

80. J.F. Zhang, Y.H. Li, X. Zhang, Cosmological constraints on neutrinos after BICEP2. Eur. Phys. J. C 74, 2954 (2014)

81. J.F. Zhang, J.J. Geng, X. Zhang, Neutrinos and dark energy after Planck and BICEP2: data consistency tests and cosmological parameter constraints. JCAP 1410(10), 044 (2014)

82. N. Palanque-Delabrouille et al., Constraint on neutrino masses from SDSS-III/BOSS Ly $\alpha$ forest and other cosmological probes. JCAP 1502(02), 045 (2015)

83. C.Q. Geng, C.C. Lee, J.L. Shen, Matter power spectra in viable $f(R)$ gravity models with massive neutrinos. Phys. Lett. B 740, 285 (2015)

84. Y.H. Li, J.F. Zhang, X. Zhang, Probing $f(R)$ cosmology with sterile neutrinos via measurements of scale-dependent growth rate of structure. Phys. Lett. B 744, 213 (2015)

85. P.A.R. Ade et al., [Planck Collaboration], Planck 2015 results. XIII. cosmological parameters, Astron. Astrophys. 594, A13 (2016)

86. J.F. Zhang, M.M. Zhao, Y.H. Li, X. Zhang, Neutrinos in the holographic dark energy model: constraints from latest measurements of expansion history and growth of structure. JCAP 1504, 038 (2015)

87. C.Q. Geng, C.C. Lee, R. Myrzakulov, M. Sami, E.N. Saridakis, Observational constraints on varying neutrino-mass cosmology. JCAP 1601(01), 049 (2016)

88. Y. Chen, L. Xu, Galaxy clustering, CMB and supernova data constraints on $\varphi \mathrm{CDM}$ model with massive neutrinos. Phys. Lett. B 752, 66 (2016)
89. R. Allison, P. Caucal, E. Calabrese, J. Dunkley, T. Louis, Towards a cosmological neutrino mass detection. Phys. Rev. D 92(12), $123535(2015)$

90. A.J. Cuesta, V. Niro, L. Verde, Neutrino mass limits: robust information from the power spectrum of galaxy surveys. Phys. Dark Univ. 13, 77 (2016)

91. Y. Chen, B. Ratra, M. Biesiada, S. Li, Z.H. Zhu, Constraints on non-flat cosmologies with massive neutrinos after Planck 2015. Astrophys. J. 829(2), 61 (2016)

92. M. Moresco, R. Jimenez, L. Verde, A. Cimatti, L. Pozzetti, C. Maraston, D. Thomas, Constraining the time evolution of dark energy, curvature and neutrino properties with cosmic chronometers. JCAP 1612(12), 039 (2016)

93. J. Lu, M. Liu, Y. Wu, Y. Wang, W. Yang, Cosmic constraint on massive neutrinos in viable $f(R)$ gravity with producing $\Lambda \mathrm{CDM}$ background expansion. Eur. Phys. J. C 76(12), 679 (2016)

94. S. Kumar, R.C. Nunes, Probing the interaction between dark matter and dark energy in the presence of massive neutrinos. Phys. Rev. D 94(12), 123511 (2016)

95. L. Xu, Q.G. Huang, Detecting the neutrinos mass hierarchy from cosmological data. Sci. China Phys. Mech. Astron. 61(3), 039521 (2018)

96. S. Vagnozzi, E. Giusarma, O. Mena, K. Freese, M. Gerbino, S. Ho, M. Lattanzi, Unveiling $v$ secrets with cosmological data: neutrino masses and mass hierarchy. Phys. Rev. D 96(12), 123503 (2017)

97. X. Zhang, Weighing neutrinos in dynamical dark energy models. Sci. China Phys. Mech. Astron. 60(6), 060431 (2017)

98. C.S. Lorenz, E. Calabrese, D. Alonso, Distinguishing between neutrinos and time-varying dark energy through cosmic time. Phys. Rev. D 96(4), 043510 (2017)

99. M.M. Zhao, J.F. Zhang, X. Zhang, Measuring growth index in a universe with massive neutrinos: a revisit of the general relativity test with the latest observations. Phys. Lett. B 779, 473 (2018)

100. S. Vagnozzi, S. Dhawan, M. Gerbino, K. Freese, A. Goobar, O. Mena, Constraints on the sum of the neutrino masses in dynamical dark energy models with $w(z) \geq-1$ are tighter than those obtained in $\Lambda$ CDM. arXiv:1801.08553 [astro-ph.CO]

101. L.F. Wang, X.N. Zhang, J.F. Zhang, X. Zhang, Impacts of gravitational-wave standard siren observation of the Einstein Telescope on weighing neutrinos in cosmology. Phys. Lett. B 782, 87 (2018)

102. E.K. Li, H. Zhang, M. Du, Z.H. Zhou, L. Xu, Probing the neutrino mass hierarchy beyond $\Lambda \mathrm{CDM}$ model. JCAP 1808, 042 (2018). https://doi.org/10.1088/1475-7516/2018/08/ 042. arXiv:1703.01554 [astro-ph.CO]

103. S. Wang, Y.F. Wang, D.M. Xia, Constraints on the sum of neutrino masses using cosmological data including the latest extended Baryon Oscillation Spectroscopic Survey DR14 quasar sample. Chin. Phys. C 42(6), 065103 (2018). https://doi.org/10.1088/ 1674-1137/42/6/065103. arXiv:1707.00588 [astro-ph.CO]

104. L. Feng, J.F. Zhang, X. Zhang, Search for sterile neutrinos in a universe of vacuum energy interacting with cold dark matter. Phys. Dark Univ. 100261 [Phys. Dark Univ. 23, 100261 (2019)]. https:// doi.org/10.1016/j.dark.2018.100261. arXiv:1712.03148 [astroph.CO]

105. M.M. Zhao, Y.H. Li, J.F. Zhang, X. Zhang, Constraining neutrino mass and extra relativistic degrees of freedom in dynamical dark energy models using Planck 2015 data in combination with low-redshift cosmological probes: basic extensions to ? CDM cosmology. Mon. Not. R. Astron. Soc. 469(2), 1713 (2017). https:// doi.org/10.1093/mnras/stx978. arXiv:1608.01219 [astro-ph.CO]

106. X. Zhang, Impacts of dark energy on weighing neutrinos after Planck 2015. Phys. Rev. D 93(8), 083011 (2016). https://doi.org/ 10.1103/PhysRevD.93.083011. arXiv:1511.02651 [astro-ph.CO]

107. Q.G. Huang, K. Wang, S. Wang, Constraints on the neutrino mass and mass hierarchy from cosmological observations. 
Eur. Phys. J. C 76(9), 489 (2016). https://doi.org/10.1140/epjc/ s10052-016-4334-z. arXiv:1512.05899 [astro-ph.CO]

108. S. Wang, Y.F. Wang, D.M. Xia, X. Zhang, Impacts of dark energy on weighing neutrinos: mass hierarchies considered. Phys. Rev. D 94(8), 083519 (2016). https://doi.org/10.1103/PhysRevD.94. 083519. arXiv:1608.00672 [astro-ph.CO]

109. S. Vagnozzi, Cosmological searches for the neutrino mass scale and mass ordering. arXiv:1907.08010 [astro-ph.CO]

110. S. Vagnozzi, Weigh them all! Cosmological searches for the neutrino mass scale and mass ordering

111. E. Giusarma, M. Gerbino, O. Mena, S. Vagnozzi, S. Ho, K. Freese, Improvement of cosmological neutrino mass bounds. Phys. Rev. D 94(8), 083522 (2016). https://doi.org/10.1103/PhysRevD.94. 083522. arXiv:1605.04320 [astro-ph.CO]

112. Z. Liu, H. Miao, Neutrino mass and mass hierarchy in various dark energy, arXiv:2002.05563 [astro-ph.CO]

113. S.R. Choudhury, S. Choubey, Updated bounds on sum of neutrino masses in various cosmological scenarios. JCAP 1809, 017 (2018)

114. R. Allahverdi, Y. Gao, B. Knockel, S. Shalgar, Indirect signals from solar dark matter annihilation to long-lived right-handed neutrinos. Phys. Rev. D 95(7), 075001 (2017). https://doi.org/10. 1103/PhysRevD.95.075001. arXiv:1612.03110 [hep-ph]

115. J. Han, R. Wang, W. Wang, X.N. Wei, Neutrino mass matrices with one texture equality and one vanishing neutrino mass. Phys. Rev. D 96(7), 075043 (2017). https://doi.org/10.1103/PhysRevD. 96.075043. arXiv:1705.05725 [hep-ph]

116. X.Y.Zhou, J.H. He, Weighing neutrinos in $f(R)$ gravity in light of BICEP2. Commun. Theor. Phys. 62, 102 (2014). https://doi.org/ 10.1088/0253-6102/62/1/18. arXiv:1406.6822 [astro-ph.CO]

117. Y.Huo, T. Li, Y. Liao, D.V. Nanopoulos, Y. Qi, Constraints on neutrino velocities revisited. Phys. Rev. D 85, 034022034022 (2012). https://doi.org/10.1103/PhysRevD.85.034022. arXiv:1112.0264 [hep-ph]

118. J.F. Zhang, B. Wang, X. Zhang, Forecast for weighing neutrinos in cosmology with SKA. Sci. China Phys. Mech. Astron. 63(8), 280411 (2020). https://doi.org/10.1007/ s11433-019-1516-y. arXiv:1907.00179 [astro-ph.CO]

119. A.D. Rivero, V. Miranda, C. Dvorkin, Observable predictions for massive-neutrino cosmologies with model-independent dark energy. Phys. Rev. D 100(6), 063504 (2019). https://doi.org/10. 1103/PhysRevD.100.063504. arXiv:1903.03125 [astro-ph.CO]

120. W. Yang, E. Di Valentino, O. Mena, S. Pan, Phys. Rev. D 102, 023535 (2020)

121. W. Yang, E. Di Valentino, O. Mena, S. Pan, R.C. Nunes, Phys. Rev. D 101, 083509 (2020)

122. W. Yang, S. Pan, R.C. Nunes, D.F. Mota, JCAP 04, 008 (2020)

123. R.Y. Guo, Y.H. Li, J.F. Zhang, X. Zhang, Weighing neutrinos in the scenario of vacuum energy interacting with cold dark matter: application of the parameterized post-Friedmann approach. JCAP 1705, 040 (2017). https://doi.org/10.1088/1475-7516/2017/05/ 040. arXiv:1702.04189 [astro-ph.CO]

124. L. Feng, D.Z. He, H.L. Li, J.F. Zhang, X. Zhang, Constraints on active and sterile neutrinos in an interacting dark energy cosmology. arXiv:1910.03872 [astro-ph.CO]

125. R.Y. Guo, J.F. Zhang, X. Zhang, Exploring neutrino mass and mass hierarchy in the scenario of vacuum energy interacting with cold dark matte. Chin. Phys. C 42(9), 095103 (2018). https://doi. org/10.1088/1674-1137/42/9/095103. arXiv:1803.06910 [astroph.CO]

126. L. Feng, H.L. Li, J.F. Zhang, X. Zhang, Exploring neutrino mass and mass hierarchy in interacting dark energy models. Sci. China Phys. Mech. Astron. 63(2), 220401 (2020). https://doi.org/10. 1007/s11433-019-9431-9. arXiv:1903.08848 [astro-ph.CO]

127. L. Amendola, Coupled quintessence. Phys. Rev. D 62, 043511 (2000). https://doi.org/10.1103/PhysRevD.62.043511. arXiv:astro-ph/9908023
128. L. Amendola, D. Tocchini-Valentini, Baryon bias and structure formation in an accelerating universe. Phys. Rev. D 66, 043528 (2002). https://doi.org/10.1103/PhysRevD.66.043528. arXiv:astro-ph/0111535

129. D. Comelli, M. Pietroni, A. Riotto, Dark energy and dark matter. Phys. Lett. B 571, 115 (2003). https://doi.org/10.1016/j.physletb. 2003.05.006. arXiv:hep-ph/0302080

130. R.G. Cai, A. Wang, Cosmology with interaction between phantom dark energy and dark matter and the coincidence problem. JCAP 0503, 002 (2005). https://doi.org/10.1088/1475-7516/2005/03/ 002. arXiv:hep-th/0411025

131. X. Zhang, Coupled quintessence in a power-law case and the cosmic coincidence problem. Mod. Phys. Lett. A 20, 2575 (2005). https://doi.org/10.1142/S0217732305017597. arXiv:astro-ph/0503072

132. W. Zimdahl, Interacting dark energy and cosmological equations of state. Int. J. Mod. Phys. D 14, 2319 (2005). https://doi.org/10. 1142/S0218271805007784. arXiv:gr-qc/0505056

133. X. Zhang, F.Q. Wu, J. Zhang, A New generalized Chaplygin gas as a scheme for unification of dark energy and dark matter. JCAP 0601, 003 (2006). https://doi.org/10.1088/1475-7516/2006/01/ 003. arXiv:astro-ph/0411221

134. B. Wang, J. Zang, C.Y. Lin, E. Abdalla, S. Micheletti, Interacting dark energy and dark matter: observational constraints from cosmological parameters. Nucl. Phys. B 778, 69 (2007). https://doi. org/10.1016/j.nuclphysb.2007.04.037. arXiv:astro-ph/0607126

135. Z.K. Guo, N. Ohta, S. Tsujikawa, Probing the coupling between dark components of the universe. Phys. Rev. D 76, 023508 (2007). https://doi.org/10.1103/PhysRevD.76.023508. arXiv:astro-ph/0702015

136. O. Bertolami, F.G. Pedro, M. Le Delliou, Dark energy-dark matter interaction and the violation of the equivalence principle from the Abell Cluster A586. Phys. Lett. B 654, 165 (2007). https://doi. org/10.1016/j.physletb.2007.08.046. arXiv:astro-ph/0703462

137. J. Zhang, H. Liu, X. Zhang, Statefinder diagnosis for the interacting model of holographic dark energy. Phys. Lett. B 659, 26 (2008). https://doi.org/10.1016/j.physletb.2007.10.086. arXiv:0705.4145 [astro-ph]

138. C.G. Boehmer, G. Caldera-Cabral, R. Lazkoz, R. Maartens, Dynamics of dark energy with a coupling to dark matter. Phys. Rev. D 78, 023505 (2008). https://doi.org/10.1103/PhysRevD.78. 023505. arXiv:0801.1565 [gr-qc]

139. J. Valiviita, E. Majerotto, R. Maartens, Instability in interacting dark energy and dark matter fluids. JCAP $\mathbf{0 8 0 7}$, 020 (2008). https://doi.org/10.1088/1475-7516/2008/07/020. arXiv:0804.0232 [astro-ph]

140. J.H. He, B. Wang, Effects of the interaction between dark energy and dark matter on cosmological parameters. JCAP 0806, 010 (2008). https://doi.org/10.1088/1475-7516/2008/06/ 010. arXiv:0801.4233 [astro-ph]

141. J.H. He, B. Wang, Y.P. Jing, Effects of dark sectors' mutual interaction on the growth of structures. JCAP 0907, 030 (2009). https:// doi.org/10.1088/1475-7516/2009/07/030. arXiv:0902.0660 [grqc]

142. J.H. He, B. Wang, P. Zhang, The imprint of the interaction between dark sectors in large scale cosmic microwave background anisotropies. Phys. Rev. D 80, 063530 (2009). https://doi.org/10. 1103/PhysRevD.80.063530. arXiv:0906.0677 [gr-qc]

143. K. Koyama, R. Maartens, Y.S. Song, Velocities as a probe of dark sector interactions. JCAP 0910, 017 (2009). https://doi.org/10. 1088/1475-7516/2009/10/017. arXiv:0907.2126 [astro-ph.CO]

144. J.Q. Xia, Constraint on coupled dark energy models from observations. Phys. Rev. D 80, 103514 (2009). https://doi.org/10.1103/ PhysRevD.80.103514. arXiv:0911.4820 [astro-ph.CO]

145. M. Li, X.D. Li, S. Wang, Y. Wang, X. Zhang, Probing interaction and spatial curvature in the holographic dark energy model. JCAP 
0912, 014 (2009). https://doi.org/10.1088/1475-7516/2009/12/ 014. arXiv:0910.3855 [astro-ph.CO]

146. L. Zhang, J. Cui, J. Zhang, X. Zhang, Interacting model of new agegraphic dark energy: cosmological evolution and statefinder diagnostic. Int. J. Mod. Phys. D 19, 21 (2010). https://doi.org/10. 1142/S0218271810016245. arXiv:0911.2838 [astro-ph.CO]

147. H. Wei, Cosmological Constraints on the Sign-Changeable Interactions. Commun. Theor. Phys. 56, 972 (2011). https://doi.org/ 10.1088/0253-6102/56/5/29. arXiv:1010.1074 [gr-qc]

148. Y. Li, J. Ma, J. Cui, Z. Wang, X. Zhang, Interacting model of new agegraphic dark energy: observational constraints and age problem. Sci. China Phys. Mech. Astron. 54, 1367 (2011). https:// doi.org/10.1007/s11433-011-4382-1. arXiv:1011.6122 [astroph.CO]

149. J.H. He, B. Wang, E. Abdalla, Testing the interaction between dark energy and dark matter via latest observations. Phys. Rev. D 83, 063515 (2011). https://doi.org/10.1103/PhysRevD.83. 063515. arXiv:1012.3904 [astro-ph.CO]

150. Y.H. Li, X. Zhang, Running coupling: does the coupling between dark energy and dark matter change sign during the cosmological evolution? Eur. Phys. J. C 71, 1700 (2011). https://doi.org/10. 1140/epjc/s10052-011-1700-8. arXiv:1103.3185 [astro-ph.CO]

151. T.F. Fu, J.F. Zhang, J.Q. Chen, X. Zhang, Holographic Ricci dark energy: interacting model and cosmological constraints. Eur. Phys. J. C 72, 1932 (2012). https://doi.org/10.1140/epjc/ s10052-012-1932-2. arXiv:1112.2350 [astro-ph.CO]

152. Z. Zhang, S. Li, X.D. Li, X. Zhang, M. Li, Revisit of the interaction between holographic dark energy and dark matter. JCAP 1206, 009 (2012). https://doi.org/10.1088/1475-7516/2012/06/ 009. arXiv:1204.6135 [astro-ph.CO]

153. J. Zhang, L. Zhao, X. Zhang, Revisiting the interacting model of new agegraphic dark energy. Sci. China Phys. Mech. Astron. 57, 387 (2014). https://doi.org/10.1007/s11433-013-5378-9. arXiv:1306.1289 [astro-ph.CO]

154. Y.H. Li, X. Zhang, Large-scale stable interacting dark energy model: cosmological perturbations and observational constraints. Phys. Rev. D 89(8), 083009 (2014). https://doi.org/10.1103/ PhysRevD.89.083009. arXiv:1312.6328 [astro-ph.CO]

155. J.J. Geng, Y.H. Li, J.F. Zhang, X. Zhang, Redshift drift exploration for interacting dark energy. Eur. Phys. J. C 75(8), 356 (2015). https://doi.org/10.1140/epjc/s10052-015-3581-8. arXiv:1501.03874 [astro-ph.CO]

156. J.L. Cui, L. Yin, L.F. Wang, Y.H. Li, X. Zhang, A closer look at interacting dark energy with statefinder hierarchy and growth rate of structure. JCAP 1509, 024 (2015). https://doi.org/10.1088/ 1475-7516/2015/09/024. arXiv:1503.08948 [astro-ph.CO]

157. R. Murgia, S. Gariazzo, N. Fornengo, Constraints on the coupling between dark energy and dark matter from CMB data. JCAP 1604, 014 (2016). https://doi.org/10.1088/1475-7516/2016/04/ 014. arXiv: 1602.01765 [astro-ph.CO]

158. B. Wang, E. Abdalla, F. Atrio-Barandela, D. Pavon, Dark matter and dark energy interactions: theoretical challenges, cosmological implications and observational signatures. Rep. Prog. Phys. 79(9), 096901 (2016). https://doi.org/10.1088/0034-4885/79/9/ 096901. arXiv:1603.08299 [astro-ph.CO]

159. A. Pourtsidou, T. Tram, Reconciling CMB and structure growth measurements with dark energy interactions. Phys. Rev. D 94(4), 043518 (2016). https://doi.org/10.1103/PhysRevD.94.043518. arXiv:1604.04222 [astro-ph.CO]

160. A.A. Costa, X.D. Xu, B. Wang, E. Abdalla, Constraints on interacting dark energy models from Planck 2015 and redshift-space distortion data. JCAP 1701, 028 (2017). https://doi.org/10.1088/ 1475-7516/2017/01/028. arXiv:1605.04138 [astro-ph.CO]

161. C. Wetterich, Phys. Lett. B 655, 201 (2007). arXiv:0706.4427 [hep-ph]
162. L. Amendola, M. Baldi, C. Wetterich, Phys. Rev. D 78, 023015 (2008). arXiv:0706.3064 [astro-ph]

163. L. Feng, X. Zhang, Revisit of the interacting holographic dark energy model after Planck 2015. JCAP 1608, 072 (2016). https:// doi.org/10.1088/1475-7516/2016/08/072 . arXiv:1607.05567 [astro-ph.CO]

164. D.M. Xia, S. Wang, Constraining interacting dark energy models with latest cosmological observations. Mon. Not. R. Astron. Soc. 463(1), 952 (2016). https://doi.org/10.1093/mnras/stw2073. arXiv:1608.04545 [astro-ph.CO]

165. C. van de Bruck, J. Mifsud, J. Morrice, Testing coupled dark energy models with their cosmological background evolution. Phys. Rev. D 95(4), 043513 (2017). https://doi.org/10.1103/ PhysRevD.95.043513. arXiv:1609.09855 [astro-ph.CO]

166. S. Kumar, R.C. Nunes, Echo of interactions in the dark sector. Phys. Rev. D 96(10), 103511 (2017). https://doi.org/10.1103/ PhysRevD.96.103511. arXiv:1702.02143 [astro-ph.CO]

167. R. Fardon, A.E. Nelson, N. Weiner, JCAP 0410, 005 (2004)

168. A.W. Brookfield, C. van de Bruck, D.F. Mota, D. TocchiniValentini, Phys. Rev. Lett. 96, 061301 (2006)

169. C. Wetterich, Nucl. Phys. B 302, 668 (1988)

170. X.-J. Bi, P. Gu, X. Wang, X. Zhang, Phys. Rev. D 69, 113007 (2004)

171. P. Hung, H. Pas, arXiv:astro-ph/0311131

172. D.B. Kaplan, A.E. Nelson, N. Weiner, Phys. Rev. Lett. 93, 091801 (2004)

173. R.D. Peccei, Phys. Rev. D 71, 023527 (2005)

174. E.I. Guendelman, A.B. Kaganovich, arXiv:hep-th/0411188

175. X. Bi, B. Feng, H. Li, X. Zhang, arXiv:hep-ph/0412002

176. V. Barger, P. Huber, D. Marfatia, arXiv:hep-ph/0502196

177. M. Cirelli, M.C. Gonzalez-Garcia, C. Pena-Garay, arXivhep$\mathrm{ph} / 0503028$

178. S. Perlmutter, G. Aldering, G. Goldhaber et al., Measurements of and from 42 high-redshift supernovae. ApJ 517, 565 (1999). arXiv:astroph/9812133

179. D.M. Scolnic et al., The complete light-curve sample of spectroscopically confirmed type Ia supernovae from Pan-STARRS1 and cosmological constraints from the combined pantheon sample. ApJ 859, 101 (2018). arXiv: 1710.00845

180. J.R. Bond, G. Efstathiou, M. Tegmark, Mon. Not. R. Astron. Soc. 291, L33-L41 (1997)

181. Y. Wang, P. Mukherjee, ApJ 650, 1 (2006)

182. E. Komatsu et al., ApJS 192, 18 (2011)

183. E. Komatsu et al., Astrophys. J. Suppl. 180, 306-329 (2009)

184. D.J. Eisenstein et al., ApJ 633, 560 (2005)

185. N. Aghanim [Planck Collaboration], Planck, et al., Results (VI, Cosmological Parameters, 2018). arXiv:1807.06209 [astroph.CO]

186. Yu. Hai, B. Ratra, F.-Y. Wang, ApJ 856, 3 (2018)

187. G. Mangano, G. Miele, S. Pastor, T. Pinto, O. Pisanti, P.D. Serpico, MNRAS 446, 2205 (2005)

188. F.N. Chamings, A. Avgoustidis, E.J. Copeland, A.M. Green, B. Li, Phys. Rev. D 100, 043525 (2019)

189. W. Yang, E. Di Valentino, O. Mena, S. Pan, Phys. Rev. D 102, 023535 (2020)

190. L. Amendola, M. Baldi, C. Wetterich, Phys. Rev. D 78, 023015 (2008) 